\title{
Can stress trigger Parkinson's disease?
}

\author{
Atbin Djamshidian, Andrew J Lees
}

- Additional material is published online only. To view please visit the journal online (http://dx.doi.org/10.1136/ jnnp-2013-305911)

The National Hospital for Neurology and Neurosurgery, Queen Square and the Reta Lila Weston Institute of Neurological Studies, UCL, London, UK

\section{Correspondence to} Professor Andrew Lees, Reta Lila Weston Institute of Neurological Studies, UCL Institute of Neurology, 1 Wakefield Street, London WC1N 1PJ, UK: andrew.lees@ucl.ac.uk

Received 21 May 2013 Revised 21 October 2013 Accepted 29 October 2013 Published Online First 20 November 2013
To cite: Djamshidian A, Lees AJ. J Neurol Neurosurg Psychiatry 2014;85: 879-882.

\section{ABSTRACT}

In this manuscript we summarize the role of chronic stress as a potential trigger factor for Parkinson's disease. Underlying mechanisms and stress-induced changes to the neuronal networks have been highlighted. Examples of stress induced reversible symptoms that resemble parkinsonism in humans and in animal models raise the question whether emotional stress can cause striatal degeneration in susceptible patients. A Pubmed literature review searching for the terms 'Stress', 'Distress and Parkinson's disease', 'Emotional Distress and Parkinson's disease', 'Stress and Parkinson's disease', 'Prodromal Parkinson's disease', 'Non motor symptoms and Parkinson's disease', 'Paradoxical kinesia', 'Psychogenic parkinsonism', 'Functional somatic syndromes', 'Chronic fatigue syndrome', 'Irritable bowel syndrome', 'Fibromyalgia', 'Dopamine and fibromyalgia', 'Dopamine and chronic fatigue syndrome' and 'Dopamine and irritable bowel syndrome' was carried out until April 2013. Articles were also identified through searches of the authors' own files. Only papers published in English were reviewed. The final reference list was generated on the basis of originality and relevance to the broad scope of this viewpoint.

\section{INTRODUCTION}

Can emotional stress trigger Parkinson's disease (PD)? This idea is compelling but not new. More than 100 years ago, Gowers wrote that prolonged anxiety and emotional shock are 'the most common antecedents of Parkinson's disease' and advised his patients to refrain from 'all causes of mental strain and of physical exhaustion'. Extreme psychological stress, such as the holocaust, has been associated with $\mathrm{PD}^{1}$ and in some studies ex-prisoners of war had a significantly higher incidence of developing PD several decades after their release. $^{2}$ More recently, several authors have speculated about the role of emotional stress in PD. ${ }^{3-5}$

Here, we hypothesise that chronic stress can cause nigrostriatal degeneration in susceptible individuals, who have impairment in stress coping mechanisms. Further, we speculate that the nonmotor symptoms in the prodromal phase of PD resemble those seen in patients with functional somatic syndromes. We emphasise that we are putting forward a theory for consideration and not claiming the evidence is definitive.

We define stress according to the Oxford English Dictionary as 'a state of mental or emotional strain or tension resulting from adverse or demanding circumstances'. ${ }^{6}$

Short self-limiting stressors evoke 'fight and flight' catecholaminergic reactions, via the sympathetic nervous system, and can be beneficial. ${ }^{7}$
Chronic stress, on the other hand, either due to major life events or due to minor but frequent 'irritations and frustrations' causes a prolonged activation of the hypothalamic-pituitary-adrenal (HPA) ${ }^{8}$ axis and triggers 'alleostatic load', a failure of adequate coping mechanisms to reduce stress. ${ }^{9}$ This can cause 'lifestyle diseases' such as type 2 diabetes mellitus, gastric ulcers or hypertension. ${ }^{10}$

A convincing link between chronic stress and neurodegeneration has been now established in patients with Alzheimer's disease. Emotionally stressed patients have a $2.7 \%$ higher risk of developing the disease and stressed dementia patients have a more rapid disease progression. ${ }^{11}$

A recent case report suggested that major stress may have triggered PD in a young woman ${ }^{12}$ and stress-induced striatal damage, with subsequent worsening of motor symptoms has been also found in several animal models of PD.

Elevated glucocorticoid levels in rodent models worsened motor performance and higher corticosterone levels led to a greater permanent loss of nigral neurons. ${ }^{4}$ 6-Hydroxydopamine (6-OHDA)-lesioned rats moved more slowly, froze more often and became rigid when challenged with stressors but reverted to normal when left alone in their home cage. ${ }^{13}$ Foot shock, tail pinch or other stressors have all been shown to increase striatal dopamine release and turnover in rodents ${ }^{14}$ and it has been suggested that this could excite striatal dopamine nerve terminals to death through increased oxidative stress. ${ }^{15}$

Chronic stress can lead to reduced dopaminergic activity within the ventral tegmental area in rodents ${ }^{16}$ and cause increased cortisol levels, and higher salivary cortisol levels compared with matched controls have been described in PD patients. ${ }^{17}$ In rats, chronic stress significantly decreased dopamine levels in the frontal cortex, striatum and the hippocampus. ${ }^{18}$

It is possible, however, that proneness to emotional stress might be the first sign of a neurodegenerative disease and changes in prefrontal, parietal or limbic networks lead to an impairment in coping with stressful life events.

\section{Possible mechanisms underlying stress-induced neuronal degeneration}

1. Stress reduces regulatory T-lymphocytes by $50 \%$ in patients who suffered from posttraumatic stress disorder ${ }^{19}$ and a similar profound reduction has been found in $\mathrm{PD}^{20}$ Dysfunction of regulatory T-lymphocytes might contribute to dopaminergic cell loss and vaccination in animal models of PD with these regulatory lymphocytes can attenuate nigrostriatal degeneration. ${ }^{21}$ 
2. Chronic stress has been also found to induce the proinflammatory networks of cytokines and chemokines, which in turn activate the HPA axis. ${ }^{22}$ Upregulation of cytokines has been linked with sudden death after major emotional trauma $^{23}$ and increased concentrations of tumour necrosis factor, interleukins and $\beta 2$ microglobulin have been reported in the substantia nigra of PD patients. ${ }^{24}$ Further, dysregulation of the HPA axis can cause dendritic remodelling, dysfunction of neurogenesis, apoptosis in hippocampal neurons and result in increased oxidative stress. ${ }^{11}$

3. Catecholamines such as dopamine are inert when stored in vesicles, but it is possible that in susceptible patients chronic stress shifts catecholamines into the cytosol where they become toxic via auto-oxidation. Oxidation of cathecholamines leads to quinones which can cause lipid peroxidation and membrane disruption ${ }^{25}$ and might ultimately cause neurodegeneration. Support for this hypothesis comes from additional preclinical studies, which have shown that chronic stress induces oxidative stress and increased protein and lipid peroxidation. $^{26}$

\section{Can emotional stress cause symptoms that resemble transient PD?}

Sayings such as 'shaking in fear', 'staring in disbelief' or 'frozen with fear' exist in many languages and suggest that acute emotional stress can mimic some features of PD.

Dramatic examples of reversible parkinsonism were frequently reported among the casualties of 'shell shock' and 'war neurosis' that in Germany were referred to as 'Kriegszitterer' ('War tremblers'). In the surviving cine films, most of these handicaps resembled a psychogenic movement disorder and suggestion therapy led to an improvement in most cases. ${ }^{27}$

A few cases, however, closely resembled PD (see online supplementary video) and neurologists described some World War I soldiers as follows: "The eyes were wide open and had a pained vacant stare. He cannot move his legs, which are rigid [...]. As in many of these patients the sole of the foot is shuffled along the ground. Another form of tremor which is coarser and less rapid than the preceding, viz. 5-6 per sec, is that which resembles paralysis agitans." ${ }^{28}$ Others observed similar findings reporting "balance is maintained with difficulty since the trunk is flexed anteriorly and the legs are partially bent at the knees. The arms hang low and stiffly at the sides, giving a simian appearance to the whole posture. Coarse tremors develop in the hands and legs (...), the facies are mask-like without expression." 29

One of us (AJL) has looked after two women who were diagnosed with PD for several years, who were not depressed and who both fully recovered following the resolution of chronic stress. Both patients had asymmetrical bradykinesia and rigidity and their motor handicaps were not distractible. Onset in both cases was gradual and one patient was found to have a mildly abnormal fluorodopa positron emission tomography scan and responded well to L-dopa (see handwriting copies in figures 1

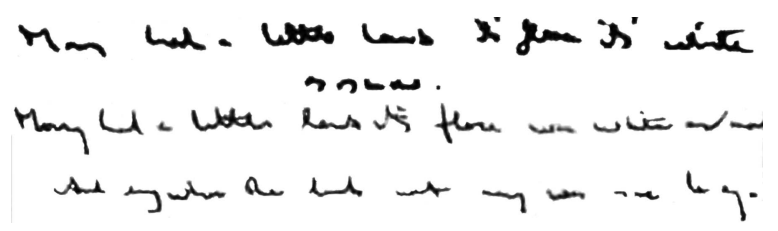

Figure 1 Small handwriting of a patient who was diagnosed with Parkinson's disease and who responded well to L-dopa.

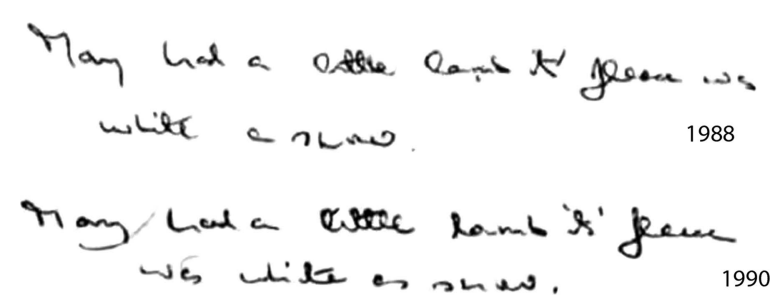

Figure 2 Same patient without any dopaminergic therapy showed significant improvement in handwriting after chronic stress has been resolved.

and 2). Once the chronic stressor disappeared, it was possible to discontinue dopaminergic medication in both patients with full and sustained recovery; iatrogenic, toxic or infectious causes were not identified in either case and there was no history of the use of neuroleptic or complementary therapies known to cause parkinsonism. One of the patients described here had no tremor and in the other case it was mild and could not be entrained.

It is, however, possible that these two patients both suffered from psychogenic parkinsonism.

Psychogenic parkinsonism is rare and it can occasionally be difficult to distinguish these cases from idiopathic PD. Characteristic features of psychogenic parkinsonism include abnormally slow and effortful movements without sequencing effect and normal speed of movement when distracted, exaggerated response to postural stability testing and an abrupt onset of the tremor with variable frequency. ${ }^{30}$ Although emotional stress often plays a precipitating role in maintaining psychogenic parkinsonism, many of these patients also have a somatoform or conversion disorder. ${ }^{30}$

These observations emphasise that prolonged chronic stress can induce a clinical picture closely resembling transient parkinsonism in susceptible individuals.

\section{The phenomenon of kinesia paradoxica}

"In acute life threatening incidents such as earthquakes ${ }^{31}$ or shipwrecks, PD patients can rarely, but spectacularly, override their often severe motor handicap." We distinguish these striking cases induced by acute severe stressors from visual, musical and proprioceptive cueing improvements in gait. The following mechanisms have been postulated to explain paradoxical kinesia in PD:

1. Acute severe stress can lead to norepinephrine activation, which then increases alertness and attention and might result in improved motor function. ${ }^{32}$ Consistent with this, rats treated with haloperidol and showing motor impairments were able to overcome their motor deficits by stress-induced norepinephrine activation. ${ }^{32}$

2. Activation of basal ganglia reserves due to fear can also overcome bradykinesia. For example, PD patients off medication speed up in a decision-making task in an attempt to avoid painful electric shocks. ${ }^{33}$

While under these acute stressors motor performance improves, chronic anxiety and stress characteristically aggravate motor symptoms in PD, particularly rest tremor. ${ }^{16}$

\section{Premotor PD}

Onset of PD is gradual and it is often difficult to determine when motor dysfunction begins. Ray Kennedy, the Arsenal and Liverpool English International football player described prominent mental and physical fatigue at least 14 years before the 
diagnosis of PD was made at the age of 35 . He also described slow thinking, a general lack of energy and disturbances of temperature regulation. "I realised that nobody had my after match routine. Usually the adrenaline is still pumping and most of the lads would be talking about what happened on the pitch, grabbing a coke or chicken leg. They were always doing something-all except me. I used to slump hunched in my seat too tired to talk or move." 34

Non-specific non-motor symptoms can sometimes lead to the misdiagnoses of a functional disorder, especially in younger patients and may delay the correct diagnosis of PD for several decades. ${ }^{35}$ In fact, non-motor complaints were the initial presentation of $21 \%$ of pathologically proven PD. ${ }^{36}$ In this cohort, depression as a prodrome of PD was found in $2.5 \%$ with $25 \%$ developing these symptoms within the first 2 years. ${ }^{36}$

Recent papers have attempted to retrospectively examine the nature and frequency of these non-specific features before the diagnosis of PD. PD patients complained of more non-specific symptoms 10 years prior to the diagnosis of $\mathrm{PD}$ and visited their general practitioners (GPs) significantly more often than controls. ${ }^{37} 38$ A significantly higher frequency of medically unexplained symptoms has been associated with PD (7-7.5\%) and PD patients with dementia (12-19\%) than in other neurodegenerative diseases such as atypical parkinsonism or the primary dementias (0-3\%). Musculoskeletal symptoms such as frozen shoulder was the initial complain in $8 \%$ of patients who developed PD up to 2 years later. ${ }^{39}$ Further, fibromyalgia, diarrhoea and autonomic dysfunction were also more frequently seen in pre-PD patients than controls. ${ }^{37}$ These results were in line with another study finding that hyposmia, constipation, disturbed sleep, depression, apathy, moodiness and increased sweating were the most frequently reported symptoms in the decade prior to the diagnosis of PD. ${ }^{40}$

One prospective study did not find a link between some psychosocial risk factors, such as major life events in childhood, major life events in adult life and the risk of PD. However, the authors speculated that other stressors such as accumulation of life events in work life, economic hardship and being somewhat socially isolated may be a risk factor for developing PD. Further, vital exhaustion defined as a psychological response reflecting a breakdown of the adaption to stress, including symptoms such as unusual fatigue, was significantly associated with the risk of developing PD. ${ }^{41}$

\section{CLINICAL OVERLAP BETWEEN FUNCTIONAL SOMATIC SYMPTOMS AND NON-MOTOR SYMPTOMS IN PD}

There is a striking overlap between the symptoms of chronic fatigue syndrome, fibromyalgia and irritable bowel syndrome, all classed as functional somatic syndromes (see online supplementary material) and the spectrum of non-motor symptoms seen in PD.

Mental fatigue, a typical feature of patients with chronic fatigue syndrome, can be found in up to $70 \%$ of PD patients at some stage of the illness. ${ }^{42}$

Gastrointestinal dysfunction has been described as the most common non-motor dysfunction in $\mathrm{PD}^{43}$ and typical features of irritable bowel syndrome such as abdominal bloating occur commonly in PD while abdominal colic can also occur. ${ }^{44}$ The longterm follow-up studies of the Honolulu-Asia Aging Study showed that constipation, a variant of irritable bowel syndrome associated with more abdominal pain and bloating than the diarrhoea variant, ${ }^{45}$ was associated with a higher risk of developing PD. ${ }^{46}$ Further, those who had constipation without PD had significantly lower substantia nigra neuronal densities and had more incidental
Lewy body pathology in the substantia nigra. ${ }^{47}$ Braak and colleagues based on neuroanatomical studies in which they used Lewy bodies as a surrogate marker for nerve cell dysfunction have claimed that PD may begin much earlier in the enteric nervous system, sympathetic ganglia, olfactory bulb and medulla oblongata. ${ }^{48}$ Indeed, $\alpha$-synuclein in colonic biopsy was found 2-5 years prior to the onset of motor symptoms ${ }^{49}$ and Lewy neurites have been also found on routine colonoscopies in 72\% of PD patients, which correlated significantly with disease progression. ${ }^{50}$

Conversely, symptoms commonly reported in PD have been found in patients with somatic syndromes. Over $75 \%$ of patients with chronic fatigue complain of 'slow thinking', 51 which can be an early feature of PD often referred to as bradyphrenia. Reduction in stride length, slowness of movements, impairment of flexing and bending knees and hips and trouble keeping up on treadmills can occur in patients with chronic fatigue syndrome. ${ }^{52}$

However, we do not suggest that chronic fatigue syndrome is a variant of $\mathrm{PD}$, although there may be some overlap at a neural level in the mechanism of symptom expression. In contrast to $\mathrm{PD}$, which is a chronic progressive degenerative disease with neuronal cell loss, chronic fatigue syndrome can be reversible with treatments such as cognitive behavioural therapy. ${ }^{53} 54$

Altered muscle synergy found in electromyography studies leading to stiffness and muscular fatigue has been also reported in patients with fibromyalgia, possibly due to striatal dysfunction. ${ }^{55}$ Restless legs syndrome, muscle and joint stiffness are frequently seen in patients with fibromyalgia. ${ }^{55}$ Tremor, stiffness and poor coordination $^{56}$ and volumetric changes within the striatum have been reported. ${ }^{55}$ Musculoskeletal pain, unusual pelvic and rectal discomfort, poor sleep and fatigue, features characteristic for functional somatic syndromes, are common in PD. ${ }^{57}$ Depression is commonly seen in functional somatic syndromes ${ }^{58}$ and $\mathrm{PD}^{57}$ and can be triggered by emotional stress. ${ }^{3}$ Whether depression can contribute to the onset of PD is, however, unclear. ${ }^{3}$

Links between dopaminergic dysfunction and medically unexplained symptoms have been described (for further details, see online supplementary material).

\section{CONCLUSION}

We speculate that chronic emotional stress may cause dopaminergic cell loss in susceptible individuals and propose that functional somatic syndromes are commonly seen in patients with PD. Dopaminergic dysfunction with abnormalities in striato-thalamocortical brain circuits may be the shared underlying cause.

\section{Implications for future research}

Hypersensitivity to stress is thought to be genetically determined and can result in alterations of DNA methylation, which in turn regulates $\alpha$-synuclein expression. ${ }^{60}$ Significant shortening of leucocyte telomeres has been found in individuals with a stressful life ${ }^{61}$ and shorter telomeres have been linked with PD in some ${ }^{62}$ but not all studies. ${ }^{63}$

Screening PD patients and patients with functional somatic syndromes for polymorphisms that are known to be important for stress regulation might prove to be one important future line of epigenetic research. Prospective long-term follow-up studies in the middle-aged population measuring stress hormones such as cortisol levels from hair, which has shown to be a valid biomarker for measuring the long-term cortisol secretion, and inflammatory markers such as T-lymphocytes is another potential line of future research that may further clarify the role of stress in PD. Finally, the roles of physical exercise and cognitive behavioural therapy in chronic stress reduction are worthy of further scientific examination. 
Acknowledgements We owe a huge debt of gratitude to Dr Alan Carson for his valuable advice. We also thank Professor Simon Wessely from the Maudsley Hospital for his helpful comments.

Contributors $A J L$ and $A D$ were equally involved in conception and design, or analysis and interpretation of data, drafting the article or revising it critically for important intellectual content and final approval of the version to be published.

Funding Reta Lila Weston Institute of Neurological Studies.

Competing interests None.

Provenance and peer review Not commissioned; externally peer reviewed.

\section{REFERENCES}

1 Salganik I, Korczyn A. Risk factors for dementia in Parkinson's disease. Adv Neurol 1990:53:343-7.

2 Gibberd FB, Simmonds JP. Neurological disease in ex-Far-East prisoners of war. Lancet 1980;2:135-7.

3 Hemmerle AM, Herman JP, Seroogy KB. Stress, depression and Parkinson's disease. Exp Neurol 2012;233:79-86.

4 Smith LK, Jadavii NM, Colwell KL, et al. Stress accelerates neural degeneration and exaggerates motor symptoms in a rat model of Parkinson's disease. Eur J Neurosci 2008:27:2133-46.

5 Smith AD, Castro SL, Zigmond MJ. Stress-induced Parkinson's disease: a working hypothesis. Physiol Behav 2002;77:527-31.

6 OED. Definition of stress. The Oxford English Dictionary. OED Online. Oxford University Press, 2013.

7 Segerstrom SC, Miller GE. Psychological stress and the human immune system: a meta-analytic study of 30 years of inquiry. Psychol Bull 2004;130:601-30.

8 McEwen BS. Physiology and neurobiology of stress and adaptation: central role of the brain. Physiol Rev 2007;87:873-904.

9 McEwen BS. Mood disorders and allostatic load. Biol Psychiatry 2003;54:200-7.

10 Liu J, Mori A. Stress, aging, and brain oxidative damage. Neurochem Res 1999:24:1479-97.

11 Rothman SM, Mattson MP. Adverse stress, hippocampal networks, and Alzheimer's disease. Neuromolecular Med 2010;12:56-70.

12 Zou K, Guo W, Tang G, et al. A Case of early onset Parkinson's disease after major stress. Neuropsychiatr Dis Treat 2013;9:1067-9.

13 Snyder AM, Stricker EM, Zigmond MJ. Stress-induced neurological impairments in an animal model of parkinsonism. Ann Neurol 1985;18:544-51.

14 Pei Q, Zetterstrom T, Fillenz M. Tail pinch-induced changes in the turnover and release of dopamine and 5-hydroxytryptamine in different brain regions of the rat. Neuroscience 1990:35:133-8.

15 Hastings TG, Lewis DA, Zigmond MJ. Role of oxidation in the neurotoxic effects of intrastriatal dopamine injections. Proc Natl Acad Sci USA 1996:93:1956-61.

16 Moore H, Rose HJ, Grace AA. Chronic cold stress reduces the spontaneous activity of ventral tegmental dopamine neurons. Neuropsychopharmacology 2001;24:410-19.

17 Djamshidian A, O'Sullivan SS, Papadopoulos A, et al. Salivary cortisol levels in Parkinson's disease and its correlation to risk behaviour. J Neurol Neurosurg Psychiatry 2011;82:1107-11.

18 Rasheed N, Ahmad A, Pandey CP, et al. Differential response of central dopaminergic system in acute and chronic unpredictable stress models in rats. Neurochem Res 2009;35:22-32.

19 Sommershof $A$, Aichinger $H$, Engler $H$, et al. Substantial reduction of naive and regulatory T cells following traumatic stress. Brain Behav Immun 2009;23:1117-24.

20 Baba Y, Kuroiwa A, Uitti RJ, et al. Alterations of T-lymphocyte populations in Parkinson disease. Parkinsonism Relat Disord 2005;11:493-8.

21 Reynolds AD, Stone DK, Hutter JA, et al. Regulatory T cells attenuate Th17 cell-mediated nigrostriatal dopaminergic neurodegeneration in a model of Parkinson's disease. J Immunol 2010;184:2261-71.

22 Haddad JJ, Saade NE, Safieh-Garabedian B. Cytokines and neuro-immune-endocrine interactions: a role for the hypothalamic-pituitary-adrenal revolving axis. J Neuroimmunol 2002;133:1-19.

23 Steptoe A, Brydon L. Emotional triggering of cardiac events. Neurosci Biobehav Rev 2009:33:63-70.

24 Hirsch EC, Hunot S. Neuroinflammation in Parkinson's disease: a target for neuroprotection? Lancet Neurol 2009;8:382-97.

25 Goldstein DS. Stress, allostatic load, catecholamines, and other neurotransmitters in neurodegenerative diseases. Endocr Regul 2011;45:91-8.

26 Lucca G, Comim CM, Valvassori SS, et al. Effects of chronic mild stress on the oxidative parameters in the rat brain. Neurochem Int 2009;54:358-62.

27 Linden SC, Jones E, Lees AJ. Shell shock at Queen Square: Lewis Yealland 100 years on. Brain 2013;136(Pt 6):1976-88.

28 Mott W. War Neuroses and Shell Shock. Oxford Medical Publications, 1919

29 Ginker RR, Spiegel JP. War neurosis in North Africa, The Tunisian Campaign January to May 1943. New York: Josiah Macy Foundation, 1943.

30 Jankovic J. Diagnosis and treatment of psychogenic parkinsonism. J Neurol Neurosurg Psychiatry 2011;82:1300-3.
31 Bonanni L, Thomas A, Onofrj M. Paradoxical kinesia in parkinsonian patients surviving earthquake. Mov Disord 2010;25:1302-4

32 Schlesinger I, Erikh I, Yarnitsky D. Paradoxical kinesia at war. Mov Disord 2007;22:2394-7.

33 Shiner $T$, Seymour B, Symmonds $M$, et al. The effect of motivation on movement: a study of bradykinesia in Parkinson's disease. PLOS ONE 2012;7:e47138.

34 Lees AJ. When did Ray Kennedy's Parkinson's disease begin? Mov Disord 1992:7:110-16

35 Ling $H$, Braschinsky M, Taba $P$, et al. Decades of delayed diagnosis in 4 levodopa-responsive young-onset monogenetic parkinsonism patients. Mov Disord 2011;26:1337-40.

36 O'Sullivan SS, Williams DR, Gallagher DA, et al. Nonmotor symptoms as presenting complaints in Parkinson's disease: a clinicopathological study. Mov Disord 2008;23:101-6.

37 Gonera EG, van't Hof M, Berger HJ, et al. Symptoms and duration of the prodroma phase in Parkinson's disease. Mov Disord 1997;12:871-6.

38 Onofrj M, Thomas A, Tiraboschi $\mathrm{P}$, et al. Updates on Somatoform Disorders (SFMD) in Parkinson's Disease and Dementia with Lewy Bodies and discussion of phenomenology. J Neurol Sci 2011;310:166-71.

39 Riley D, Lang AE, Blair RD, et al. Frozen shoulder and other shoulder disturbances in Parkinson's disease. J Neurol Neurosurg Psychiatry 1989;52:63-6.

40 Gaenslen A, Swid I, Liepelt-Scarfone I, et al. The patients' perception of prodromal symptoms before the initial diagnosis of Parkinson's disease. Mov Disord 2011;26:653-8.

41 Clark AJ, Ritz B, Prescott $\mathrm{E}$, et al. Psychosocial risk factors, pre-motor symptoms and first-time hospitalization with Parkinson's disease: a prospective cohort study. Eur J Neurol 2013:20:1113-20.

42 Friedman JH, Abrantes A, Sweet LH. Fatigue in Parkinson's disease. Expert Opin Pharmacother 2011:12:1999-2007.

43 Pfeiffer RF. Gastrointestinal dysfunction in Parkinson's disease. Lancet Neurol 2003:2:107-16

44 Edwards LL, Pfeiffer RF, Quigley EM, et al. Gastrointestinal symptoms in Parkinson's disease. Mov Disord 1991;6:151-6.

45 Talley NJ, Dennis EH, Schettler-Duncan VA, et al. Overlapping upper and lower gastrointestinal symptoms in irritable bowel syndrome patients with constipation or diarrhea. Am J Gastroenterol 2003:98:2454-9.

46 Abbott RD, Petrovitch $H$, White LR, et al. Frequency of bowel movements and the future risk of Parkinson's disease. Neurology 2001;57:456-62.

47 Petrovitch $\mathrm{H}$, Abbott RD, Ross GW, et al. Bowel movement frequency in late-life and substantia nigra neuron density at death. Mov Disord 2009;24:371-6.

48 Braak H, Del Tredici K, Rub U, et al. Staging of brain pathology related to sporadic Parkinson's disease. Neurobiol Aging 2003;24:197-211.

49 Shannon KM, Keshavarzian A, Dodiya HB, et al. Is alpha-synuclein in the colon a biomarker for premotor Parkinson's disease? Evidence from 3 cases. Mov Disord 2012:27:716-19.

50 Lebouvier T, Neunlist M, Bruley des Varannes S, et al. Colonic biopsies to assess the neuropathology of Parkinson's disease and its relationship with symptoms. PLoS ONE 2010;5:e12728.

51 Lane TJ, Manu P, Matthews DA. Depression and somatization in the chronic fatigue syndrome. Am J Med 1991;91:335-44.

52 Boda WL, Natelson BH, Sisto SA, et al. Gait abnormalities in chronic fatigue syndrome. J Neurol Sci 1995;131:156-61.

53 Baker R, Shaw EJ. Diagnosis and management of chronic fatigue syndrome or myalgic encephalomyelitis (or encephalopathy): summary of NICE guidance. BMJ 2007;335:446-8

54 Price JR, Mitchell E, Tidy E, et al. Cognitive behaviour therapy for chronic fatigue syndrome in adults. Cochrane Database Syst Rev 2008;(3):CD001027.

55 Schmidt-Wilcke $T$, Luerding R, Weigand $T$, et al. Striatal grey matter increase in patients suffering from fibromyalgia-a voxel-based morphometry study. Pain 2007;132(Suppl 1):S109-16.

56 Wolfe F, Smythe HA, Yunus MB, et al. The American College of Rheumatology 1990 Criteria for the classification of fibromyalgia. Report of the Multicenter Criteria Committee. Arthritis Rheum 1990:33:160-72.

57 Gallagher DA, Lees AJ, Schrag A. What are the most important nonmotor symptoms in patients with Parkinson's disease and are we missing them? Mov Disord 2010;25:2493-500.

58 Wessely S, Nimnuan C, Sharpe M. Functional somatic syndromes: one or many? Lancet 1999;354:936-9.

59 Gilad GM, Gilad VH. Strain, stress, neurodegeneration and longevity. Mech Ageing Dev 1995;78:75-83.

60 Babenko 0, Kovalchuk I, Metz GA. Epigenetic programming of neurodegenerative diseases by an adverse environment. Brain research 2012;1444:96-111.

61 Epel ES, Blackburn EH, Lin J, et al. Accelerated telomere shortening in response to life stress. Proc Natl Acad Sci USA 2004;101:17312-15.

62 Maeda T, Guan JZ, Koyanagi M, et al. Aging-associated alteration of telomere length and subtelomeric status in female patients with Parkinson's disease. Neurogenet 2012;26:245-51.

63 Eerola J, Kananen L, Manninen K, et al. No evidence for shorter leukocyte telomere length in Parkinson's disease patients. J Gerontol A Biol Sci Med Sci 2010;65:1181-4. 